\title{
Sensitivity of high strain rate of structural elements in relation to dynamics properties of material
}

\author{
Leopold Kruszka $^{1, a}$, Iurii S. Vorobiov ${ }^{2}$, and Nataliia Iu. Ovcharova ${ }^{2}$ \\ ${ }^{1}$ The Gen. Jaroslaw Dabrowski Military University of Technology, 2 Gen. S. Kaliski St., Warsaw 00-908, Poland \\ ${ }^{2}$ A.N. Podgorny Institute of Mechanical Engineering Problems, National Ukrainian Academy of Sciences, 2/10 D. \\ Pozharskoho St., Kharkov 61046, Ukraine
}

\begin{abstract}
Protective structures such as process chambers, protective boxes, facing elements of vehicles, personal protection equipment, motors cases etc. widely used in modern technology has been tested in the following project. Under the influence of impact loads, the three-dimensional dynamic stress-strain state with finite dynamic displacements and deformations has arised. The deformations occur in the elastic- plastic stage. In the analysis of the high-rate deformations, the dynamic properties of the materials, which are determined based on experimental data should be taken into account. The dynamic stress state of structural elements depends essentially on the dynamic properties of the materials used. The problem is solved using the finite element method, which takes into account the specifics of the process. Boundary conditions in the element nodes must satisfy the equality movement as well as derivatives. The formed function allows to describe continuous and smooth stress changes. The numerical analysis of the dynamic stress-strain state of structural elements under impact loads, takes into account different dynamic properties of the materials. A series of numerical calculations allows to reveal the features of high-rate deformation elements of protective structures and makes recommendations to improve their dynamic strength under different loading conditions.
\end{abstract}

\section{Introduction and problem statement}

Analysis of the dynamic stress-strain state of a number of modern structures, such as input and output devices, cases of gas turbine engines, safety boxes, process chambers, lining elements of vehicles, personal protective elements of energy systems, aerospace, transport and military equipment, under the influence of local shock and impulse loads is an actual and complex problem [16]. Identification of danger zones localization of stresses in impact places allows to find ways to reduce their level and increase the dynamic stability of elements of critical structures. This requires the construction of refined mathematical models. Together with intense impact loads, three dimensional dynamic stress-strain state develops in the elastic-plastic stage. Strength properties of most materials change depending on the value and rate of deformation. Therefore three-dimensional models with the dynamic properties of the material, which are determined on the basis of experimental studies are used [1-7]. In different papers different approaches and methods of accounting of the dynamic properties of materials are used. Known constitutive model described in [1]. A method for determining the dynamic properties of the material by a Hopkinson bar is presented in [2]. Common approaches to the modeling of the dynamic properties of the materials are included in the monographs [3,5]. Large elasticplastic deformations are investigated in [4]. The physical nature of the behavior of materials under the influence of different types of loads is considered in [6]. Influence of dynamic properties of materials in the process of high-rate

\footnotetext{
${ }^{a}$ Corresponding author: 1 kruszka@wat.edu.pl
}

deformation of structural elements is an actual and vital problem. If the equation of state reflects the elastic-plastic character of deformation and dynamic properties of the material, then the problem is physically nonlinear. Under intense loads finite displacement and deformation arises resulting in geometrical nonlinearity of problems [8].

A mathematical model for the analysis of the problem must take into account the heterogeneity of the material's structural elements as originally given as well as arisen during high-rate deformation. Therefore, all the material characteristics are variable in the spatial coordinates and time. Dependencies for stress from strains are determined based on a dynamic version of the theory of plastic deformation. Dynamic hardening of materials reflects the dependencies of the stress intensity $\sigma_{i}$ from intensities $\varepsilon \dot{i}$ of the strain and of the strain rate $\sigma_{i}=\sigma_{i}\left(\varepsilon_{i}, \dot{\varepsilon}_{i}\right)$ [7,9].

Figure 1 shows some of the dependencies of the relative dynamic elastic limit $\sigma_{s d} / \sigma_{s}$ for a number of structural materials obtained on the basis of experimental data [7]. Those curves correspond to structural materials: 1 - ЭИ 878 (X15H8A18) steel, 2 - X18H10T steel, 3 - OT41 alloy, 4 - ЭП410 (Х15H5Д2Т) steel, 5 $\mathrm{AM} 2 \mathrm{M}$ alloy.

It can be seen that the rate of deformation provides the greatest influence on steel alloys and less influence on aluminium.

In region I the experiments were carried out in a range of strain rate $8 \cdot 10^{1}-6 \cdot 10^{2} 1 / \mathrm{s}$, and in region II in the range of $1,3 \cdot 10^{2}-3,5 \cdot 10^{3} 1 / \mathrm{s}$. It is established that the equation $\sigma_{s d} / \sigma_{s}=\left[1-\left(\frac{\dot{\varepsilon}}{D}\right)^{\frac{1}{n}}\right]$ quite accurately describes the dependence of the dynamic elastic limit the strain rate. 


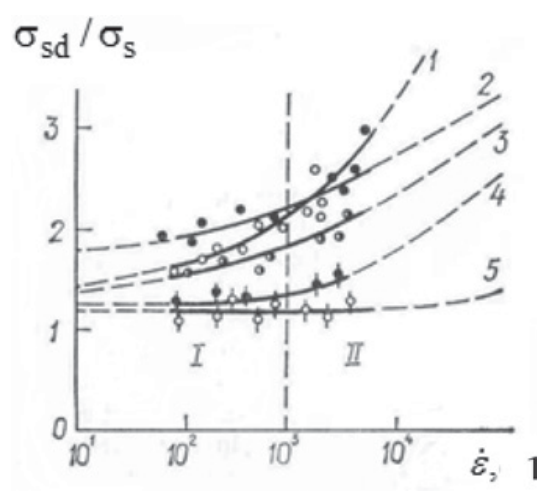

Figure 1. Experimental dependence of the dynamic elastic limit in relation to the strain rate [7].

The value of the coefficients $\mathrm{D}$ and $\mathrm{n}$ for various materials are given in [7]. In the general case strain-rate hardening of the material may be described as

$\sigma=E \varepsilon_{s}\left[1+\left(\frac{\dot{\varepsilon}_{e q}}{D}\right)^{1 / n}\right]+\sigma_{s}\left\{\frac{\varepsilon_{e q}}{\varepsilon_{s}}-\left[1+\left(\frac{\varepsilon_{e q}}{D}\right)^{1 / n}\right]\right\}^{m}$

where, $\varepsilon_{s}, D, m, n$ - characteristics of strain hardening and rate hardening of the material; $\varepsilon_{e q}, \dot{\varepsilon}_{e q}$ - the elasticplastic deformation and rate deformation of specimens measured during the tests.

The first term reflects an increase of the dynamic yield stress, and the second - large plastic deformation [7].

However, in numerical studies different dependencies for describing the dynamic properties of the material are used. Their form is determined by the way of the experimental data processing used by different authors [1-5]. It is interesting to compare the results of calculations using different, the most common dependencies.

The problem of the variant of finite element method is solved takes into account the specifics of the process. An important requirement for the choice of the type of finite elements is a accounting of all specified factors. The boundary conditions at the nodes of the elements must satisfy the equality displacements as well as derivatives. The functions formed allow to describe the continuous and smooth variation of stresses.

\section{Analysis of the numerical results}

Numerical analysis based on the finite element method allows to determine the distribution, magnitude and time variation of the dynamic displacements, strains and stresses in different types of loads [8-10]. However, the numerical results depend strongly from the method of accounting of the dynamic properties of the material. For comparison, consider the impact on the rectangular element of the steel structure with thickness $h=8 \mathrm{~mm}$, impactor mass $m=0,1 \mathrm{~kg}$ with a velocity $200 \mathrm{~m} / \mathrm{s}$ for different accounting methods of dynamic properties of the material.

Trying to solve the problem in the elastic formulation, the increase of intensity of the load leads, as expected, to overestimation of maximum stress (to $800 \mathrm{MPa}$ ) when the undervaluation of displacements equals about $0.25 \mathrm{~mm}$.

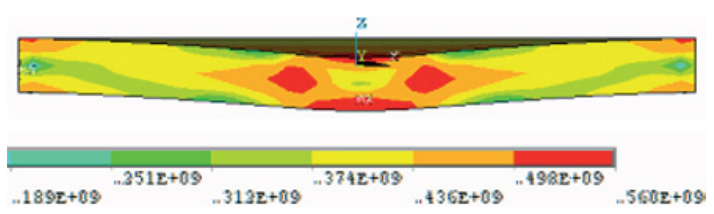

Figure 2. Distribution of equivalent stress $\sigma_{e q}$ under the influence of the impactor with the dynamic properties of the material according to the relationship (2).

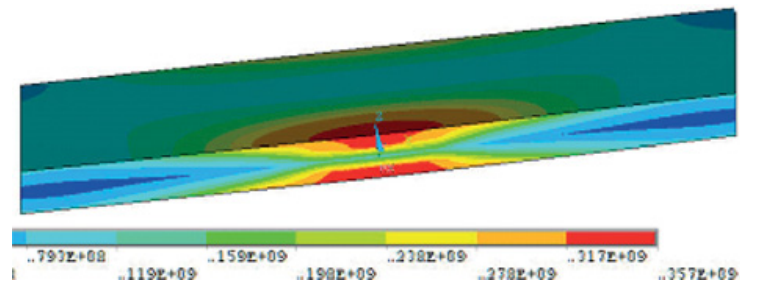

Figure 3. Distribution of equivalent stress under the influence of the impactor in a refined setting.

In [8-10] it is shown that it is appropriate to use the account of the dynamic properties of material by the Perzyna dependency [5], which is a special case of the relation (1)

$$
\sigma_{i}=\left[1+\left(\frac{\dot{\varepsilon}_{i}^{p l}}{\gamma}\right)^{m}\right] E \varepsilon_{i}
$$

where $E$ - elastic modulus;

$m$ and $r$ - strain rate sensitivity coefficients;

$\dot{\varepsilon}_{i}^{p l}-$ deformation rate at the plastic stage.

In this case a decrease in the largest equivalent stress $(500 \mathrm{MPa})$ and the increase in displacements $(2.8 \mathrm{~mm})$, besides changing the character of distribution of equivalent stresses $\sigma_{e q}$ (Fig. 2) can be noticed.

This model can be refined by taking into account finite deformations and increasing the number of degrees of freedom at the nodes. In this case, the maximum equivalent stress is further reduced $(350 \mathrm{MPa})$, and the amount of displacement is increased (Fig. 3).

Dynamic properties of the material can be addressed in some other form using Peirce's dependency [4], which uses the same notation as in the Perzyna dependency (2).

$$
\sigma_{i}=\left[1+\left(\frac{\dot{\varepsilon}_{i}^{p l}}{\gamma}\right)\right]^{m} E \varepsilon_{i}
$$

In this case finite displacement and a large number of degrees of freedom at the nodes is also taken into account. (Fig. 4).

In this case there has been some reduction of the maximum equivalent stresses $(312 \mathrm{MPa})$ and the value of maximum displacements does not change. When unloading there is a sharp reduction of stresses and redistribution of dynamic stress-strain state.

It can be noticed that during refining of the elasticplastic and dynamic properties of the material, as well as taking into account finite deformations, increasingly evident nonlinear character of deformation with increasing dynamic changes of the stress-strain state in the pipe of 

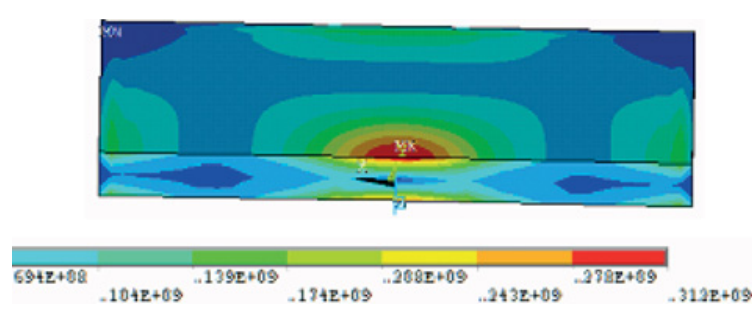

Figure 4. Distribution of equivalent stresses under the influence of a drummer in relation to the dynamic properties of the material according to the relationship (3).

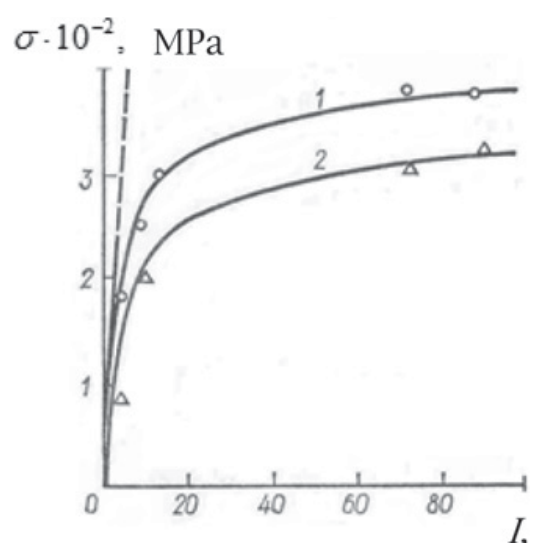

Figure 5. Change of the axial (1) and radial (2) stresses in the pipe with the increase of impact load [7].

gas pipeline with an increase in the local shock load by total momentum $I$ are presented [7].

The radius of the pipe equals as follows: $0,5 \mathrm{~m}$, thickness $0,01 \mathrm{~m}$, length $5,5 \mathrm{~m}$. In the analysis of large plastic deformations the dependence which is a special case of (1) was used. displacements and strains at lower values of stresses can be noticed. To further illustrate this phenomenon the results of numerical studies of the

$$
\sigma_{i}=A \cdot \varepsilon_{i}^{m}\left[1-\left(\frac{\dot{\varepsilon}_{i}}{D}\right)^{\frac{1}{n}}\right]
$$

where $A=1323 \mathrm{MPa}$ - hardening modulus in the plastic stage; $m=0,14 ; n=3,8 ; D=5201 / \mathrm{s}$.

Figure 5 shows a comparison of the numerical (curves) and experimental (points) results for the axial (curve 1) and radial (curve 2) stress [7]. It shows the influence of the plastic and dynamic properties of the material and the non-linear character of deformation as the load increases. The dotted line shows the limit of the elastic.

\section{Conclusions}

Dynamic properties of the material have a significant influence on the development of high-rate process of elastic-plastic deformation of structural elements under intense impact loads. Solution of the problem in the elastic formulation is possible only at low speeds and low loads, when $\left(\frac{\dot{\varepsilon}_{i}}{D}\right)^{1 / n} \ll 1$. When unloading, differences in the character of the stress-strain state can be observed, the unloading process starts at different stages of the stressstrain state.

When choosing a sufficiently appropriate elasticplastic and dynamic characteristics of the material reliable results can be achieved, which can properly evaluate the allowable impact loading or dynamic strength of structural elements. It should strive to comparison of the results obtained when using different accounting methods of dynamic properties of the material. It allows to increase the accuracy of numerical calculations. In any case, multivariate numerical studies allow to identify character of elastic-plastic high-rate deformation of structural elements and secure their dynamic strength.

\section{References}

[1] G.R. Johnson, W.H. Cook, A constitutive model and data for metals subjected to large strains high strain rates and high temperatures, Proceedings of the $7^{\text {th }}$ International Symposium on Ballistics, The Hague, The Netherlands, April 1983.

[2] L. Kruszka, W.K. Nowacki, New application of the Hopkinson pressure bar technique to determining dynamic behavior of materials, J. of Theoretical and Applied Mechanics, 2 (1996).

[3] M.A. Meyers, Dynamics behavior of materials, Wiley, New York 1994.

[4] D. Peirce and D.R.J. Owen, A model for large deformations of elastic-viscoplastic solids at finite strains: computational issues, in: Finite Inelastic Deformations: Theory and Applications, SpringerVerlag, Berlin 1992.

[5] P. Perzyna, Fundamental problems in viscoplasticity, Advances in Applied Mechanics, 9 (1968).

[6] V.T. Troshchenko, A.A. Lebedev, V.A. Strizhalo. Mechanical behaviour of materials under different types of loading (in Russian), Logos, Kiev, 2000.

[7] Iu.S. Vorobiov, A.V. Kolodyazhny, V.I. Sevryukov and E.G. Yanyutin, High-speed deformation of structure elements (in Russian), Naukova Dumka, Kiev 1989.

[8] Iu.S. Vorobiov, N.Iu. Ovcharova, L. Kruszka, Nonlinear deformations of structures cylindrical element under local shock, Proceedings of the $4^{\text {th }}$, International Conference on Nonlinear Dynamics ND-KhPI2013 - Ukraine, pp. 351-357, 2013.

[9] Iu.S. Vorobiov, L. Kruszka, N.Iu. Ovcharova, Finite Element Analysis of Local Shock Loading on Structures Cylindrical Elements, Proceedings of The $8^{\text {th }}$ International Symposium on Impact Engineering (ISIE2013), Osaka University, Japan, pp. 499-504, 2013.

[10] Iu.S. Vorobiov, N.Iu. Ovcharova, Dynamics of structural elements under impact loads (in Russian), Vibration in Engineering and Technologies, 74, (2014). 